\title{
Effect of Chemical Reaction on Mass Transfer due to a Permeable Rotating Heated Disk
}

\author{
B.R. Sharma \\ Department of Mathematics \\ Dibrugarh University
}

\author{
Hemanta Konwar \\ Research Scholar \\ Department of Mathematics \\ Dibrugarh University
}

\begin{abstract}
An unsteady three dimensional flow in an incompressible viscous, chemically reacting fluid mixture due to a permeable rotating heated disk is studied by taking into account the effects of magnetic field, ohmic heating, viscous dissipation, chemical reaction and suction or injection. The system of nonlinear partial differential equations governing the flow, heat and mass transfer is reduced to a system of nonlinear ordinary differential equations by using similarity transformations. The resulting system of ordinary coupled nonlinear differential equations is solved numerically by bvp $4 c$ and solutions are presented graphically. All numerical calculations are done with respect to air at $20^{\circ} \mathrm{C}(\mathrm{Pr}=0.71)$ in presence of Hydrogen $(\mathrm{Sc}=0.22)$. Numerical values of the local skin frictions and the rate of heat and mass transfers are shown in tables.
\end{abstract}

\section{Keywords}

Unsteady flow, Rotating Disk, Magnetic effect, Viscous dissipation, Ohmic heating, Chemical reaction.

\section{INTRODUCTION}

Flow due to a rotating disk is one of the classical problems of fluid mechanics and has several applications in engineering and industries such as rotating machinery like computer storage devices, gas turbine rotors, centrifugal pumps, rotational viscometers and some aerodynamic related problems. The first solution to the problem of rotating disk flow was obtained by von Karman (1921). The solution was further improved by Cochran (1934). Wagner (1948) discussed heat transfer from a rotating disk to ambient air. Millsaps and Pohlhausen (1952) discussed heat transfer by laminar flow from a rotating plate. Benton (1966) improved Cochran's solutions and solved the unsteady problem. Finding the importance of suction or injection Kuiken(1971) studied the effect of normal blowing on the flow near a rotating disk of infinite extent. Georges (1989) numerically studied convective heat transfer over a rotating rough disk with uniform wall temperature. Cheng and Lin (1994) discussed unsteady and steady mass transfer by laminar forced flow against a rotating disk. Hassan and Attia (1997) investigated the steady magneto hydro dynamics boundary layer flow due to an infinite disk rotating with uniform angular velocity in presence of the Hall current. Alam and Sattar (1998) considered the unsteady MHD free convection and mass transfer flow in a rotating system with thermal diffusion. Attia (1998) studied the unsteady MHD flow near a rotating porous disk with uniform suction or injection. Takhar et al. (2002) carried out study on the unsteady MHD flow and heat transfer on a rotating disk in an ambient fluid. Maleque and Sattar (2003) further studied the transient convective flow due to a rotating disc with magnetic field and heat absorption effects.
Attia (2006) continued study on the unsteady flow and heat transfer of viscous incompressible fluid with temperature dependent viscosity due to a rotating disc in a porous medium. Osalusi et al. (2007) studied the effects of Ohmic heating and viscous dissipation on unsteady MHD and slip flow over a porous rotating disk. Sharma and Singh (2010) discussed thermal diffusion in a binary fluid mixture flow due to a rotating disk of uniform high suction in presence of a weak axial magnetic field. Sibanda and Makinde (2010) investigated steady MHD flow and heat transfer past a rotating disk in a porous medium with ohmic heating and viscous dissipation. Maleque (2010) studied Dufour and Soret effects on unsteady MHD convective heat and mass transfer flow due to a rotating disk. Mustafa (2012) considered MHD fluid flow and heat transfer due to a stretching rotating disk. Khidir (2013) considered the effect of viscous dissipation, ohmic heating and radiation on MHD flow past a rotating disk embedded in a porous medium with variable properties.

It was found that none of the above mentioned authors took into account the effects of chemical reaction and its order. Therefore present authors have the curiosity to investigate the effect of chemical reaction and its order on unsteady flow due to a permeable rotating heated disk.

Chemical reaction have several industrial applications such as food processing, cooling of nuclear reactors, underground energy transport, magnetized plasma flow, polymer production, manufacturing of ceramic and enhanced oil recovery. Mass transfer proceeds as long as there is a difference in concentrations of some chemical species in the mixture. Chemical reaction processes can be classified into two broad categories namely homogeneous and heterogeneous. A chemical reaction is said to be homogeneous reaction if it occurs uniformly throughout a given phase and heterogeneous if it takes place in a restricted area or within the boundary of a phase. A chemical reaction is said to be of order $\mathrm{n}$ if the rate of reaction is proportional to the nth power of concentration. The order of the chemical reaction depends on several factors. One of the simplest chemical reactions is the first order reaction in which the rate of reaction is directly proportional to the species concentration. Second and third order chemical reactions are usually destructive in nature.

The aim of this paper is to investigate the effect of chemical reaction and its order on mass transfer due to a permeable rotating heated disk in presence of the magnetic field, ohmic heating, viscous dissipation. The system of non-linear partial differential equations governing the unsteady flow, heat and mass transfer is first converted in to a system of ordinary differential equations by using suitable transformations and then solved by using bvp4c. 


\section{FORMULATION OF THE PROBLEM}

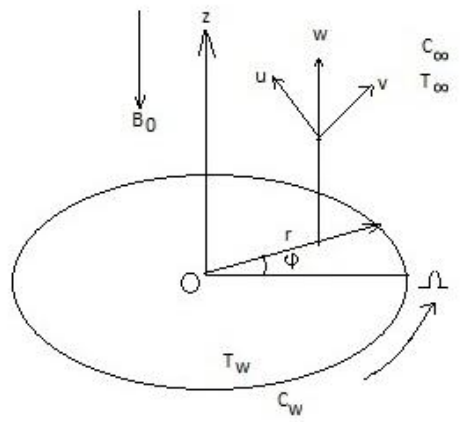

Fig1. Physical model and co-ordinate system

Consider an infinite disk rotating about $\mathrm{z}$ axis which passes through $r=0$ with a uniform angular velocity $\Omega$ in a viscous incompressible, electrically conducting and chemically reacting fluid mixture. $\mathrm{z}$ is the vertical axis with $\mathrm{r}$ as the radial and $\varphi$ as the tangential axes respectively in cylindrical polar coordinate system $(r, \varphi, z)$. Let $(\mathrm{u}, \mathrm{v}, \mathrm{w})$ are the components of velocity in the increasing directions of $r, \varphi$ and $z$ respectively. Let the fluid mixture occupies the region $\mathrm{z}>0$. A magnetic field of strength $\mathrm{B}_{0}$ is applied normal to the plane of the disk. Let the temperature, pressure and species concentration of the fluid mixture far away from the surface of the disk are $\mathrm{T}_{\infty}, \mathrm{P}_{\infty}$ and $\mathrm{C}_{\infty}$. The surface of the disk is maintained at constant high temperature $\mathrm{T}_{\mathrm{w}}$ and constant species concentration $\mathrm{C}_{\mathrm{w}}$ such that $\mathrm{T}_{\mathrm{w}}>\mathrm{T}_{\infty}$ and $\mathrm{C}_{\mathrm{w}}>\mathrm{C}_{\infty}$.

The following assumptions are made:

1. The flow is axisymmetric and so all functions are independent of $\varphi$.

2. The fluid mixture is infinite in extent in the positive $\mathrm{z}$ direction.

3. Magnetic field has a constant magnetic flux density.

4. The magnetic Reynolds number is very small.

5. A uniform suction or injection is applied through the surface of the disk.

Under the above assumptions, the governing equations describing the conservation of mass, momentum, energy and species in cylindrical polar co-ordinates $(r, \varphi, z)$ can be written as follows:

$$
\frac{\partial \mathrm{u}}{\partial \mathrm{r}}+\frac{\mathrm{u}}{\mathrm{r}}+\frac{\partial \mathrm{w}}{\partial \mathrm{z}}=0
$$

$\frac{\partial \mathrm{u}}{\partial \mathrm{t}}+\mathrm{u} \frac{\partial \mathrm{u}}{\partial \mathrm{r}}-\frac{\mathrm{v}^{2}}{\mathrm{r}}+\mathrm{w} \frac{\partial \mathrm{u}}{\partial \mathrm{z}}=-\frac{1}{\rho} \frac{\partial \mathrm{P}}{\partial \mathrm{r}}+\mathrm{v}\left(\frac{\partial^{2} \mathrm{u}}{\partial \mathrm{r}^{2}}+\frac{1}{\mathrm{r}} \frac{\partial \mathrm{u}}{\partial \mathrm{r}}-\frac{\mathrm{u}}{\mathrm{r}^{2}}+\frac{\partial^{2} \mathrm{u}}{\partial \mathrm{z}^{2}}\right)$

$-\frac{\sigma \mathrm{B}_{0}^{2} \mathrm{u}}{\rho}$

$\frac{\partial \mathrm{v}}{\partial \mathrm{t}}+\mathrm{u} \frac{\partial \mathrm{v}}{\partial \mathrm{r}}+\frac{\mathrm{uv}}{\mathrm{r}}+\mathrm{w} \frac{\partial \mathrm{v}}{\partial \mathrm{z}}=\mathrm{v}\left(\frac{\partial^{2} \mathrm{v}}{\partial \mathrm{r}^{2}}+\frac{1}{\mathrm{r}} \frac{\partial \mathrm{v}}{\partial \mathrm{r}}-\frac{\mathrm{v}}{\mathrm{r}^{2}}+\frac{\partial^{2} \mathrm{v}}{\partial \mathrm{z}^{2}}\right)$

$-\frac{\sigma \mathrm{B}_{0}{ }^{2} \mathrm{v}}{\rho}$

$\frac{\partial \mathrm{w}}{\partial \mathrm{t}}+\mathrm{u} \frac{\partial \mathrm{w}}{\partial \mathrm{r}}+\mathrm{w} \frac{\partial \mathrm{w}}{\partial \mathrm{z}}=-\frac{1}{\rho} \frac{\partial \mathrm{P}}{\partial \mathrm{z}}+v\left(\frac{\partial^{2} \mathrm{w}}{\partial \mathrm{r}^{2}}+\frac{1}{\mathrm{r}} \frac{\partial \mathrm{w}}{\partial \mathrm{r}}+\frac{\partial^{2} \mathrm{w}}{\partial \mathrm{z}^{2}}\right)$

$$
\begin{aligned}
& \frac{\partial \mathrm{T}}{\partial \mathrm{t}}+\left(\mathrm{u} \frac{\partial \mathrm{T}}{\partial \mathrm{r}}+\mathrm{w} \frac{\partial \mathrm{T}}{\partial \mathrm{z}}\right)=\frac{\mathrm{k}}{\rho \mathrm{C}_{\mathrm{p}}}\left(\frac{\partial^{2} \mathrm{~T}}{\partial \mathrm{r}^{2}}+\frac{1}{\mathrm{r}} \frac{\partial \mathrm{T}}{\partial \mathrm{r}}+\frac{\partial^{2} \mathrm{~T}}{\partial \mathrm{z}^{2}}\right)+ \\
& \frac{\mathrm{D}_{\mathrm{m}} \mathrm{k}_{\mathrm{T}}}{\mathrm{C}_{\mathrm{S}} \mathrm{C}_{\mathrm{p}}}\left(\frac{\partial^{2} \mathrm{C}}{\partial \mathrm{r}^{2}}+\frac{1}{\mathrm{r}} \frac{\partial \mathrm{C}}{\partial \mathrm{r}}+\frac{\partial^{2} \mathrm{C}}{\partial \mathrm{z}^{2}}\right)+\frac{v}{\mathrm{C}_{\mathrm{p}}}\left[\left(\frac{\partial \mathrm{u}}{\partial \mathrm{z}}\right)^{2}+\left(\frac{\partial \mathrm{v}}{\partial \mathrm{z}}\right)^{2}\right]+ \\
& \frac{\sigma \mathrm{B}_{0}^{2}}{\rho \mathrm{C}_{\mathrm{p}}}\left(\mathrm{u}^{2}+\mathrm{v}^{2}\right) \\
& \frac{\partial \mathrm{C}}{\partial \mathrm{t}}+\left(\mathrm{u} \frac{\partial \mathrm{C}}{\partial \mathrm{r}}+\mathrm{w} \frac{\partial \mathrm{C}}{\partial \mathrm{z}}\right)=\mathrm{D}_{\mathrm{m}}\left(\frac{\partial^{2} \mathrm{C}}{\partial \mathrm{r}^{2}}+\frac{1}{\mathrm{r}} \frac{\partial \mathrm{C}}{\partial \mathrm{r}}+\frac{\partial^{2} \mathrm{C}}{\partial \mathrm{z}^{2}}\right)+ \\
& \frac{\mathrm{D}_{\mathrm{m}} \mathrm{k}_{\mathrm{T}}}{\mathrm{T}_{\mathrm{m}}}\left(\frac{\partial^{2} \mathrm{~T}}{\partial \mathrm{r}^{2}}+\frac{1}{\mathrm{r}} \frac{\partial \mathrm{T}}{\partial \mathrm{r}}+\frac{\partial^{2} \mathrm{~T}}{\partial \mathrm{z}^{2}}\right)-\mathrm{k}_{\mathrm{C}}\left(\mathrm{C}-\mathrm{C}_{\infty}\right)^{\mathrm{n}}
\end{aligned}
$$

with the boundary conditions

$\mathrm{u}=0, \mathrm{v}=\Omega \mathrm{r}, \mathrm{w}=-\mathrm{w}_{0}, \mathrm{~T}=\mathrm{T}_{\mathrm{w}}, \mathrm{C}=\mathrm{C}_{\mathrm{w}}$ at $\mathrm{z}=0$

and

$$
\mathrm{u} \rightarrow 0, \mathrm{v} \rightarrow 0, \mathrm{~T} \rightarrow \mathrm{T}_{\infty}, \mathrm{C} \rightarrow \mathrm{C}_{\infty}, \mathrm{P} \rightarrow \mathrm{P}_{\infty} \text { at } \mathrm{Z} \rightarrow \infty
$$

where $\mathrm{C}$ is the concentration of rarer and lighter component, $\mathrm{T}$ is the temperature, $\mathrm{P}$ is the pressure, $\rho$ is the density, $\mathrm{k}$ is the thermal conductivity, $\mathrm{C}_{\mathrm{s}}$ is the concentration susceptibility, $\mathrm{C}_{\mathrm{p}}$ is the specific heat at constant pressure, $\sigma$ is the electrical conductivity, $\mathrm{D}_{\mathrm{m}}$ is the molecular diffusivity, $\mathrm{k}_{\mathrm{T}}$ is the thermal diffusivity ratio, $\mathrm{T}_{\mathrm{m}}$ is the mean temperature of the fluid mixture, $\mathrm{k}_{\mathrm{C}}$ is the dimensional chemical reaction parameter and $\mathrm{n}$ is the order of chemical reaction respectively.

Introducing the following similarity variables

$$
\begin{aligned}
& \eta=\frac{z}{\delta}, u=r \Omega f^{\prime}(\eta), v=r \Omega g(\eta), w=-2 \delta \Omega f(\eta), \\
& p-p_{\infty}=\rho v \Omega P(\eta), \phi=\frac{C-C_{\infty}}{C_{w}-C_{\infty}} \text { and } \theta=\frac{T-T_{\infty}}{T_{w}-T_{\infty}}
\end{aligned}
$$

The new transformation equations are

$$
\begin{aligned}
& \mathrm{f}^{\prime \prime \prime}+\frac{\eta \delta}{v} \frac{\mathrm{d} \delta}{\mathrm{dt}} \mathrm{f}^{\prime \prime}-\mathrm{R}_{\mathrm{r}}\left[M \mathrm{Mf}^{\prime}+\left(\mathrm{f}^{\prime}\right)^{2}-\mathrm{g}^{2}-2 \mathrm{ff} \mathrm{f}^{\prime \prime}\right] \\
& \mathrm{g}^{\prime \prime}+\frac{\eta \delta}{v} \frac{\mathrm{d} \delta}{\mathrm{dt}} \mathrm{g}^{\prime}-\mathrm{R}_{\mathrm{r}}\left[2 \mathrm{f}^{\prime} \mathrm{g}-2 \mathrm{fg}^{\prime}+\mathrm{Mg}\right] \\
& \theta^{\prime \prime}+\mathrm{D}_{\mathrm{f}} \operatorname{Pr} \phi^{\prime \prime}+\operatorname{Pr} \frac{\eta \delta}{v} \frac{\mathrm{d} \delta}{\mathrm{dt}} \theta^{\prime}+2 \operatorname{PrR}_{\mathrm{r}} \mathrm{f} \theta^{\prime}+ \\
& \operatorname{Pr} \operatorname{Ec}\left\{\left(\mathrm{f}^{\prime \prime}\right)^{2}+\left(\mathrm{g}^{\prime}\right)^{2}\right\}+\operatorname{MPrEcR}_{\mathrm{r}}\left\{\left(\mathrm{f}^{\prime}\right)^{2}+(\mathrm{g})^{2}\right\}=0 \\
& \phi^{\prime \prime}+\operatorname{SrSc} \theta^{\prime \prime}+\operatorname{Sc} \frac{\eta \delta}{v} \frac{\mathrm{d} \delta}{\mathrm{dt}} \phi^{\prime}+2 \operatorname{ScR}_{\mathrm{r}} \mathrm{f} \phi^{\prime}-\gamma \phi=0
\end{aligned}
$$

where

$$
\begin{aligned}
& \mathrm{M}=\frac{\sigma \mathrm{B}_{0}^{2}}{\rho \Omega}, \operatorname{Pr}=\frac{\rho v \mathrm{C}_{\mathrm{p}}}{\mathrm{k}}, \mathrm{R}_{\mathrm{r}}=\frac{\Omega \delta^{2}}{v}, \mathrm{Sc}=\frac{v}{\mathrm{D}_{\mathrm{m}}}, \mathrm{Ec}=\frac{\mathrm{r}^{2} \Omega^{2}}{\left(\mathrm{~T}_{\mathrm{w}}-\mathrm{T}_{\infty}\right) \mathrm{C}_{\mathrm{p}}}, \\
& \mathrm{Sr}=\frac{\mathrm{D}_{\mathrm{m}} \mathrm{k}_{\mathrm{T}}\left(\mathrm{T}_{\mathrm{w}}-\mathrm{T}_{\infty}\right)}{\mathrm{T}_{\mathrm{m}}\left(\mathrm{C}_{\mathrm{w}}-\mathrm{C}_{\infty}\right) \nu}, \gamma=\frac{\mathrm{k}_{\mathrm{c}} \delta^{2}}{\mathrm{D}_{\mathrm{m}}\left(\mathrm{C}_{\mathrm{w}}-\mathrm{C}_{\infty}\right)^{\mathrm{n}-1}} \text { and } \mathrm{D}_{\mathrm{f}}=\frac{\mathrm{D}_{\mathrm{m}} \mathrm{k}_{\mathrm{T}}\left(\mathrm{C}_{\mathrm{w}}-\mathrm{C}_{\infty}\right)}{\mathrm{C}_{\mathrm{s}} \mathrm{C}_{\mathrm{p}} v\left(\mathrm{~T}_{\mathrm{w}}-\mathrm{T}_{\infty}\right)}
\end{aligned}
$$

are magnetic parameter, Prandtl number, rotational parameter, Schmidt number, Eckert number, Soret number, chemical reaction parameter and Dufour number respectively.

Above Eqs.9-12 depend on a single similarity variable $\eta$ except a time dependent term $\frac{\eta \delta}{v} \frac{\mathrm{d} \delta}{\mathrm{dt}}$ where time $\mathrm{t}$ appears 
explicitly. Thus the similarity conditions require that $\frac{\eta \delta}{\mathrm{v}} \frac{\mathrm{d} \delta}{\mathrm{dt}}$ must be a constant quantity. We therefore assume that $\frac{\eta \delta}{v} \frac{\mathrm{d} \delta}{\mathrm{dt}}$ $=$ constant $=\mathrm{A}$. This in turn gives

$\delta=\sqrt{2 \mathrm{~A} v \mathrm{t}}+\mathrm{L}$

where the constant of integration $\mathrm{L}$ is determined through the conditions that $\delta=\mathrm{L}$ when $\mathrm{t}=0$ and $\mathrm{A}=0$ implies that $\delta=\mathrm{L}$ which represent the length scale for steady flow.

If $\mathrm{A} \neq 0$ (say $\mathrm{A}=2)$ then $\delta=2 \sqrt{v \mathrm{t}}+\mathrm{L}$

represents length scale for unsteady flow and gives a class of solutions. Since $\delta$ is a scaling factor and similarity variable other values of A would not change the nature of the solution except the scale would be different.

Using the equation (14) in Eqs.(9-12) we get

$$
\begin{aligned}
& \mathrm{f}^{\prime \prime \prime}+2 \eta f^{\prime \prime}-\mathrm{R}_{\mathrm{r}}\left[M f^{\prime}+\left(\mathrm{f}^{\prime}\right)^{2}-\mathrm{g}^{2}-2 \mathrm{ff} \mathrm{f}^{\prime \prime}\right]=0 \\
& \mathrm{~g}^{\prime \prime}+2 \eta \mathrm{g}^{\prime}-\mathrm{R}_{\mathrm{r}}\left[2 \mathrm{f}^{\prime} \mathrm{g}-2 \mathrm{fg}^{\prime}+\mathrm{Mg}\right]=0 \\
& \theta^{\prime \prime}+2 \operatorname{Pr}\left(\eta+\mathrm{R}_{\mathrm{r}} \mathrm{f}\right) \theta^{\prime}+\mathrm{D}_{\mathrm{f}} \operatorname{Pr} \phi^{\prime \prime}+\operatorname{Pr} \operatorname{Ec}\left\{\left(\mathrm{f}^{\prime \prime}\right)^{2}+\left(\mathrm{g}^{\prime}\right)^{2}\right\} \\
& +\operatorname{MPr} \operatorname{Ec} \mathrm{R}_{\mathrm{r}}\left\{\left(\mathrm{f}^{\prime}\right)^{2}+(\mathrm{g})^{2}\right\}=0 \\
& \phi^{\prime \prime}+2 \operatorname{Sc}\left(\eta+\mathrm{R}_{\mathrm{r}} \mathrm{f}\right) \phi^{\prime}+\operatorname{SrSc} \theta^{\prime \prime}-\gamma \phi^{\mathrm{n}}=0
\end{aligned}
$$

together with the boundary conditions

$$
\begin{aligned}
& \mathrm{f}^{\prime}=0, \mathrm{~g}=1, \mathrm{f}=\mathrm{f}_{\mathrm{w}}, \theta=1, \phi=1 \text { when } \eta=0 \\
& \mathrm{f}^{\prime} \rightarrow 0, \mathrm{~g} \rightarrow 0, \theta \rightarrow 0, \phi \rightarrow 0 \text { when } \eta \rightarrow \infty
\end{aligned}
$$

Here prime denotes differentiation with respect to $\eta$.Uniform suction or injection is represented by $\mathrm{f}_{\mathrm{w}}=\frac{\mathrm{w}_{0}}{4 \sqrt{\mathrm{vt}} \Omega}$. The parameter $f_{w}>0$ is for suction, $f_{w}=0$ is for impermeable disk and $f_{w}<0$ is for injection. Eq. (1) is identically satisfied with the help of the above transformations.

\section{DISCUSSIONS AND RESULTS}

The concentration profiles for values of $\gamma=[0,0.5,0.8,1]$; $\mathrm{Pr}$ $=0.71 ; \mathrm{Sr}=0.5 ; \mathrm{M}=0.5 ; \mathrm{R}_{\mathrm{r}}=0.5 ; \mathrm{Sc}=0.22 ; \mathrm{Ec}=0.3 ; \mathrm{D}_{\mathrm{f}}=$ $0.2 ; \mathrm{f}_{\mathrm{w}}=1 ; \mathrm{n}=1$ is shown in the Fig. 2 .

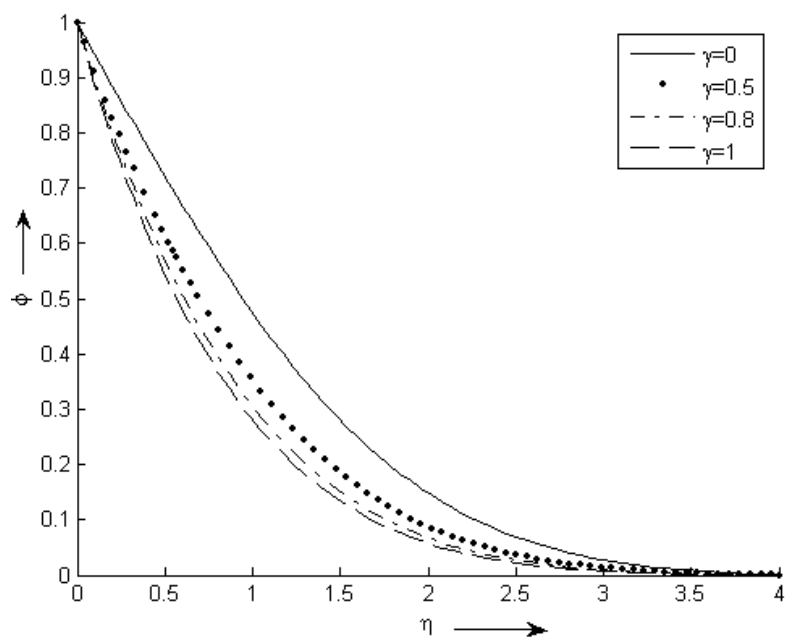

Fig 2: Concentration profiles for $\gamma$
Figs. 2 shows that the effect of increase in the values of the chemical reaction parameter $(\gamma)$ decreases the concentration of the rarer and lighter component of the fluid mixture which in turn decreases the concentration buoyancy effects of the fluid mixture.

The concentration profiles for values of $\mathrm{n}=[1,2,3], \gamma=0.5$; $\operatorname{Pr}=0.71 ; \mathrm{Sr}=0.5 ; \mathrm{M}=0.5 ; \mathrm{R}_{\mathrm{r}}=0.5 ; \mathrm{Sc}=0.22 ; \mathrm{Ec}=0.3 ; \mathrm{D}_{\mathrm{f}}$ $=0.2 ; f_{\mathrm{w}}=1$ is shown in the Fig. 3 .

Fig. 3 depicts that the effect of increase in the values of the order of chemical reaction (n) increases the concentration of the rarer and lighter component of the fluid mixture which in turn increases the concentration buoyancy effects of the fluid mixture.

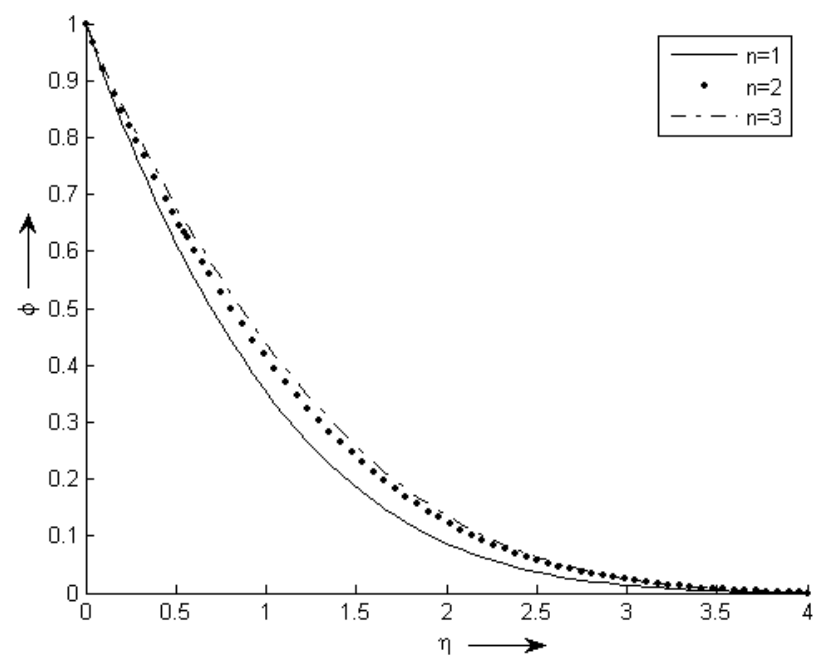

Fig 3: Concentration profiles for $\mathbf{n}$

The tangential shear stress $\left(\tau_{\mathrm{t}}\right)$, the radial shear stress $\left(\tau_{\mathrm{r}}\right)$, the heat flux $\left(\mathrm{q}_{\mathrm{w}}\right)$ and the mass flux $\left(\mathrm{M}_{\mathrm{w}}\right)$ are given by the following expressions:

$$
\begin{aligned}
& \tau_{\mathrm{t}}=\mu\left(\frac{\partial \mathrm{v}}{\partial \mathrm{z}}\right)_{\mathrm{z}=0} \propto \mathrm{g}^{\prime}(0) \\
& \tau_{\mathrm{r}}=\mu\left(\frac{\partial \mathrm{u}}{\partial \mathrm{z}}\right)_{\mathrm{z}=0} \propto \mathrm{f}^{\prime \prime}(0) \\
& \mathrm{q}_{\mathrm{w}}=-\mathrm{k}\left(\frac{\partial \mathrm{T}}{\partial \mathrm{z}}\right)_{\mathrm{z}=0} \propto-\theta^{\prime}(0)
\end{aligned}
$$

and

$$
\mathbf{M}_{\mathrm{w}}=-\mathrm{D}_{\mathrm{m}}\left(\frac{\partial \mathrm{C}}{\partial \mathrm{z}}\right)_{\mathrm{z}=0} \propto-\phi^{\prime}(0)
$$

Numerical values of the tangential, the radial skin friction coefficients, the rate of heat and mass transfer coefficients for various values of M, Ec, $\gamma$ and $f_{w}$ are shown in tables1-4. For all the tables $\mathrm{Pr}=0.71 ; \mathrm{Sr}=0.5 ; \mathrm{D}_{\mathrm{f}}=0.2 ; \mathrm{Sc}=0.22 ; \mathrm{R}_{\mathrm{r}}=0.5$; $\mathrm{n}=1$. 
Table:1

\begin{tabular}{|l|c|c|c|c|}
\hline $\mathrm{M}$ & $-\mathrm{g}^{\prime}(0)$ & $\mathrm{f}^{\prime \prime}(0)$ & $-\theta^{\prime}(0)$ & $-\phi^{\prime}(0)$ \\
\hline 0 & 1.8374 & 0.0997 & 1.1726 & 0.9400 \\
\hline 0.5 & 1.9176 & 0.0957 & 1.1550 & 0.9418 \\
\hline 1.5 & 2.0686 & 0.0889 & 1.1218 & 0.9453 \\
\hline 3 & 2.2761 & 0.0808 & 1.0763 & 0.9502 \\
\hline
\end{tabular}

Table:2

\begin{tabular}{|l|l|l|}
\multicolumn{1}{|c}{$\mathrm{M}=0.5 ; \mathrm{f}_{\mathrm{w}}=1 ; \gamma=0.5$} \\
\hline Ec & $-\theta^{\prime}(0)$ & $-\phi^{\prime}(0)$ \\
\hline 0.2 & 1.2282 & 0.9342 \\
\hline 0.5 & 1.0084 & 0.9572 \\
\hline 0.7 & 0.8619 & 0.9725 \\
\hline 1 & 0.6421 & 0.9956 \\
\hline
\end{tabular}

Table:3

$\mathrm{Ec}=0.3 ; \mathrm{M}=0.5 ; \mathrm{f}_{\mathrm{w}}=1$
\begin{tabular}{|l|l|l|}
\hline$\gamma$ & $-\theta^{\prime}(0)$ & $-\phi^{\prime}(0)$ \\
\hline 0.2 & 1.1778 & 0.7675 \\
\hline 0.4 & 1.1622 & 0.8865 \\
\hline 0.6 & 1.1480 & 0.9947 \\
\hline 0.8 & 1.1350 & 1.0943 \\
\hline
\end{tabular}

Table 4:

$\mathrm{Ec}=0.3 ; \mathrm{M}=0.5 ; \gamma=0.5$

\begin{tabular}{|l|l|l|l|l|}
\hline $\mathrm{f}_{\mathrm{w}}$ & $-\mathrm{g}^{\prime}(0)$ & $\mathrm{f}^{\prime \prime}(0)$ & $-\theta^{\prime}(0)$ & $-\phi^{\prime}(0)$ \\
\hline-1.5 & 0.5011 & 0.1551 & 0.3008 & 0.7300 \\
\hline-0.5 & 0.9429 & 0.1338 & 0.5789 & 0.8146 \\
\hline 0 & 1.2315 & 0.1205 & 0.7525 & 0.8567 \\
\hline 0.5 & 1.5585 & 0.1076 & 0.9455 & 0.8990 \\
\hline 1.5 & 2.3027 & 0.0852 & 1.3780 & 0.9855 \\
\hline
\end{tabular}

\section{CONCLUSION}

In this study, we have examined the effects of chemical reaction and its order on an unsteady flow about a permeable rotating disk in presence of magnetic field, viscous dissipation and Ohmic heating. Numerical results are obtained by using bvp4c.

From the above discussions we conclude the followings:

1. Increase in the values of the chemical reaction parameter $\gamma$ is to decrease the concentration of the rarer and lighter component of the fluid mixture.

2. Increase in the values of the order of the chemical reaction $\mathrm{n}$ is to increase the concentration of the rarer and lighter component of the fluid mixture.

\section{REFERENCES}

[1] Von Karman, T. 1921. Uber laminare und turbulente reibung, ZAMM - Journal of Applied Mathematics and Mechanics, 1(4), 233-252.

[2] Cochran, W.G. 1934. The flow due to a rotating disk, Proceedings of the Cambridge Philosophical Society, 30(3), 365-375.

[3] Wagner, C. 1948. Heat transfer from a rotating disk to ambient air, Journal of Applied Physics, 19, 837-839.

[4] Millsaps, K. and Pohlhausen, K. 1952. Heat transfer by laminar flow from a rotating plate, Journal of Aerospace Science, 19(2), 120-126.

[5] Benton, E.R. 1966. On the flow due to a rotating disk, Journal of Fluid Mechanics, 24(4), 781-800.

[6] Kuiken, H.K. 1971. The effect of normal blowing on the flow near a rotating disk of infinite extent, Journal of Fluid Mechanics., 47(4), 789-798.

[7] Georges, L.P, 1989. Numerical study of convective heat transfer over a rotating rough disk with uniform wall temperature, Int. Com. in Heat and Mass Transfer, 16(1), 107-113.

[8] Cheng, W. T. and Lin, H. T. 1994. Unsteady and steady mass transfer by laminar forced flow against a rotating disk, Heat and Mass Transfer, 30(2), 101-108.

[9] Hassan, A.L.A. and Attia, H.A. 1997. Flow due to a rotating disk with Hall effect, Physics Letters A, 228 (45), 286-290.

[10] Alam, M.M. and Sattar, M.A. 1998. Unsteady MHD Free Convection and Mass Transfer Flow in a Rotating System with Thermal Diffusion, Journal of Energy Heat and Mass Transfer, 20, 77-87.

[11] Attia, H.A. 1998. Unsteady MHD flow near a rotating porous disk with uniform suction or injection, Fluid Dynamics Research, 23, 283-290.

[12] Takhar, H.S., Singh, A.K., Nath, G. 2002. Unsteady MHD flow and heat transfer on a rotating disk in an ambient fluid, Int. Journal of Thermal Sciences, 41, 147155 .

[13] Maleque, Kh.A. and Sattar, M.A. 2003. Transient Convective Flow Due to a Rotating Disc with Magnetic Field and Heat Absorption Effects, Journal of Energy, Heat and Mass Transfer, 25, 279-291. 
[14] Attia, H.A. 2006. Unsteady flow and heat transfer of viscous incompressible fluid with temperature dependent viscosity due to a rotating disc in a porous medium, Journal of Physics and Applied Mathematics, 39, 979991.

[15] Osalusi, E., Side, J., Harris, R. 2007. The effects of Ohmic heating and viscous dissipation on unsteady MHD and slip flow over a porous rotating disk, Int. Comm. Heat Mass Transfer, 34(9-10), 1017-1029.

[16] Sharma, B.R. and Singh, R.N. 2010. Thermal diffusion in a binary fluid mixture flow due to a rotating disk of uniform high suction in presence of a weak axial magnetic field, Theoret. Appl. Mechanics, 37(3), 161187

[17] Sibanda, P. and Makinde, O.D. 2010. On steady MHD flow and heat transfer past a rotating disk in a porous medium with ohmic heating and viscous dissipation, Int. Journal of Numerical Methods Heat Fluid Flow, 20(3), 269-285.

[18] Maleque, Kh. A. 2010. Dufour and Soret effects on unsteady MHD convective heat and mass transfer flow due to a rotating disk, Latin American applied Research, 40(2), pp. 105-111.

[19] Mustafa, T. 2012. MHD fluid flow and heat transfer due to a stretching rotating disk, Int. Journal of Thermal Sciences, 51, 195-201.

[20] Ahmed, A. Khidir. 2013. Viscous dissipation, Ohmic heating and radiation effects on MHD flow past a rotating disk embedded in a porous Medium with variable properties, Arab Journal of Mathematics , 2, 263-277. 